\title{
Publishipigfuse phase transition of BST thin films in the microwave domain
}

Kevin Nadaud, ${ }^{1, \text { a) }}$ Caroline Borderon, ${ }^{2, \text { b) }}$ Raphaël Renoud, ${ }^{2}$ Areski Ghalem, ${ }^{3}$ Aurelian Crunteanu, ${ }^{3}$ Laure Huitema, ${ }^{3}$ Frédéric Dumas-Bouchiat, ${ }^{4}$ Pascal Marchet, ${ }^{4}$ Corinne Champeaux, ${ }^{4}$ and Hartmut W. Gundel ${ }^{2}$ ${ }^{1)}$ GREMAN UMR 7347, Université de Tours, CNRS, INSA-CVL, 16 rue Pierre et Marie Curie, 37071 Tours, France

${ }^{2)}$ IETR, UMR CNRS 6164, Université de Nantes, 44322 Nantes,

France

${ }^{3)}$ XLIM, UMR CNRS 7252, Université de Limoges, 123 avenue Albert Thomas, 87060 Limoges, France

${ }^{4)}$ Univ. Limoges, CNRS, IRCER, UMR 7315, 12 rue Atlantis, F- 87068 Limoges, France.

Ferroelectric materials are widely used in the paraelectric phase in order to realize tunable capacitors with reduced losses and low hysteresis effect. Nevertheless, for polycrystalline thin films, the ferro/paraelectric phase transition can be diffuse and some crystallites can exhibit a ferroelectric nature even if the sample seem to be globally in a paraelectric phase. In this case, domain wall motions are responsible for the sensitivity of the dielectric properties to the driving field and are very dissipative phenomena. In this paper, we evaluate, in the high-frequencies band, the ferroelectricity impact on the properties of BST thin films near the phase transition, by measuring the film's dielectric properties as a function of the incident RF power and for different temperatures.

Keywords: Ferroelectrics, domain walls, hyperbolic law, microwave frequency, temperature

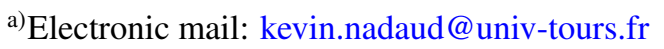

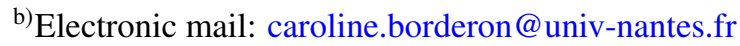


Publishing o handle the ever-increasing number of different transmission protocols at high transmission data rates, microwave devices should be more and more reconfigurable. The reconfigurability at device or sub-system levels (switches, filters, antennas,...) requires the employment of semiconductor-based or MEMS (MicroElectroMechanical Systems) components or functional materials (such as ferroelectrics) which can conveniently adapt the state of a device to a specific, required state (e.g. operating frequency, polarization $)^{1-3}$. The advantages of using ferroelectrics for tunable devices come from their large permittivity variation near the transition temperature with an applied external field, their low leakage current (since they-behave like insulators) and their very fast response time. Ferroelectric materials can be thus conveniently used to realize variable capacitors for which the capacitance values can be tuned using a static electrical field.

The dielectric characterization of the material is obviously necessary in order to integrate them into a practical device. The non-linear characterizations of permittivity and losses as a function of frequency are usually done using high driving fields ${ }^{4-6}$, for which the non-linear phenomena arise by the harmonic generation. Nevertheless, at low driving fields ${ }^{7,8}$ ferroelectric materials also exhibit a non-linear behavior due to the domain wall pinning/unpinning ${ }^{9,10}$. These non-linearities need to be taken into account for the integration of the material into a device since they are associated to very dissipative phenomena 1,12 which may considerably decrease the figure of merit of the material ${ }^{8,13}$ ( $F o M$, ratio between relative tunability and dielectric losses).

In the present paper we study the influence of the incident power and especially of the temperature on the dielectric properties of a ferroelectric thin film in the microwave range. We focus on moderate power leyels (corresponding to a low driving field $E_{A C}$ ) in order to evaluate the influence of the domain wall motion (Rayleigh region) but also on the influence of high power levels in order to study the coalescence of domains.

At low fields, called the Rayleigh region ${ }^{9}$, the polarization state of the material remains identical and thus the hyperbolic law can be applied ${ }^{10}$ :

$$
\varepsilon_{r}=\varepsilon_{r l}+\sqrt{\varepsilon_{r-r e v}^{2}+\left(\alpha_{r} E_{A C}\right)^{2}}
$$

where $\varepsilon_{r l}$ corresponds to the lattice contribution, $\varepsilon_{r-r e v}$ to the domain wall vibration and $\alpha_{r}$ to pinning/unpinning of the domain walls.

The present study is done on (100) epitaxial $\mathrm{Ba}_{2 / 3} \mathrm{Sr}_{1 / 3} \mathrm{TiO}_{3}$ (BST) thin films deposited by pulsed laser deposition on a (100)-oriented $\mathrm{MgO} / \mathrm{Ir}$ substrate. More details on the process and the structural characterization are reported elsewhere ${ }^{7,14}$. The film has a thickness of $0.45 \mu \mathrm{m}$ and it 


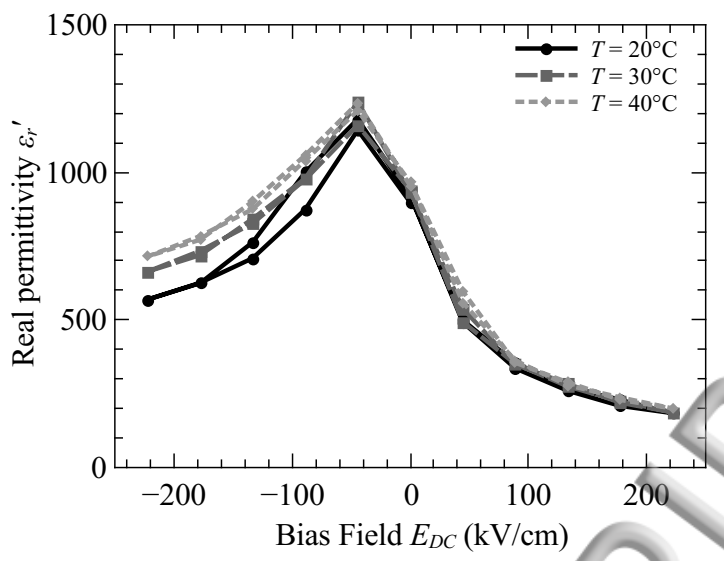

Figure 1: Relative permittivity of the BST thin film measured at $100 \mathrm{MHz}$ as a function of the bias field and for different temperatures.

consists of highly oriented columnar grains of approximately $0.1 \mu \mathrm{m}$ in width.

For a single crystal having the studied composition, the Curie temperature is close to $15^{\circ} \mathrm{C}^{15}$. To verify the Curie temperature of our polycrystalline sample, the characteristic $\varepsilon_{r}^{\prime}\left(E_{D C}\right)$ loops where measured at $100 \mathrm{MHz}$ at different temperatures (Fig. 1). At $20^{\circ} \mathrm{C}$, the cycle is slightly open for a negative bias but only one maximum is observed indicating that the material is near its Curie temperature. The loop is also very similar to the measurements made at $30^{\circ} \mathrm{C}$ and $40{ }^{\circ} \mathrm{C}$ which have very closed loops showing a material in a paraelectric state. This last point is confirmed in Ref. 16 by the piezoelectric resonances which only appear when a bias field is applied. The little shift of the Curie temperature compared to the single crystal value may be attributed to the stress into the film ${ }^{17}$. For a single crystal, the temperature of the ferro/paraelectric phase transition is well defined but in a polycristal, the size and shape distributions of the crystallites and the stress applied on the thin film can lead to a dispersion of the Curie temperature. If the overall behavior of the material seems clearly paraelectric on the $\varepsilon_{r}^{\prime}\left(E_{D C}\right)$ loop, it is necessary to study in more detail the dielectric characteristics of the samples in the transition region.

Metal-insulator-metal (MIM) capacitor has been chosen to perform the dielectric characterizations of the fabricated thin films. The advantage of this geometry is the possibility to perform measurements at low and high frequencies and the facility in extracting the material's permittivity using the well-known parallel plate expression:

$$
C=\varepsilon_{0} \varepsilon_{r}^{\prime} \frac{S}{t_{B S T}}
$$

with $\varepsilon_{0}=8.85 \times 10^{-12} \mathrm{~F} / \mathrm{m}$ the vacuum permittivity, $\varepsilon_{r}^{\prime}$ the relative permittivity of the material, $S$ 
Publishithg area of the electrode and $t_{B S T}$ the thickness of the material.

The bottom electrode of the MIM structure consists of an iridium layer $(100 \mathrm{~nm})$ while the square top electrode $\left(30 \times 30 \mu \mathrm{m}^{2}\right)$ is made of gold/titanium $(200 \mathrm{~nm} / 10 \mathrm{~nm})$. The capacitor is measured using a Cascade ground-signal-ground (GSG) probe of $125 \mu \mathrm{m}$ pitch and a vector network analyzer (VNA, Rohde \& Schwarz ZVA24) in the $50 \mathrm{MHz}-2 \mathrm{GHz}$ frequency band. The measurements have been carried out at different temperatures $\left(20^{\circ} \mathrm{C}, 30^{\circ} \mathrm{C}\right.$ and $\left.40^{\circ} \mathrm{C}\right)$. The range of temperature is imposed by our test-bench, consisting of a Peltier heat pump, which is not able to regulate the temperature outside this range.

The complex capacitance, defined as $C^{*}=C(1-j \tan \delta)$ similarly to the complex permittivity ${ }^{4}$, can be obtained using the reflection $S$-parameter $\Gamma_{C}::^{18}$

$$
C^{*}=\frac{1}{j \omega Z_{0}} \frac{1-\Gamma_{C}}{1+\Gamma_{C}}
$$

where $Z_{0}$ is the reference impedance $(50 \Omega), \omega$ the angular frequency and $j$ the imaginary unit. The capacitance and dielectric losses can thus be obtained:

$$
C=\operatorname{Re}\left[C^{*}\right], \quad \tan \delta=-\frac{\operatorname{Im}\left[C^{*}\right]}{\operatorname{Re}\left[C^{*}\right]},
$$

The power of the incident high-frequency signal has been varied from $-20 \mathrm{dBm}$ to $15 \mathrm{dBm}$ corresponding to a maximum value of the driving field $E_{A C}=80 \mathrm{kV} / \mathrm{cm}$. In addition to the reflection calibration, a power calibration has been performed and the indicated power corresponds to the real power incident on the ferroelectric capacitor.

When using a VNA, only the incident power $P_{R F}$ can be set and a conversion into the actual driving field $E_{A C}{ }^{7}$ can be done using:

$$
E_{A C}=\frac{V_{A C}}{t_{B S T}}=\left|1+\Gamma_{C}\right| \frac{\sqrt{2 Z_{0} P_{R F}}}{t_{B S T}},
$$

with $t_{B S T}$ the thickness of the thin film and $\Gamma_{C}$ the reflection coefficient of the load which can be written as:

$$
\Gamma_{C}=\frac{1-j \omega Z_{0} C^{*}}{1+j \omega Z_{0} C^{*}},
$$

where $C^{*}\left(P_{R F}\right)$ is the complex capacitance of the tunable material. For a given frequency $\omega$, the reflection coefficient depends on the capacitance $C^{*}$. As a consequence, the prefactor $\left|1+\Gamma_{C}\right|$, and thus the driving field, decrease when the frequency increases, even if the incident power is kept constant ${ }^{7}$. 


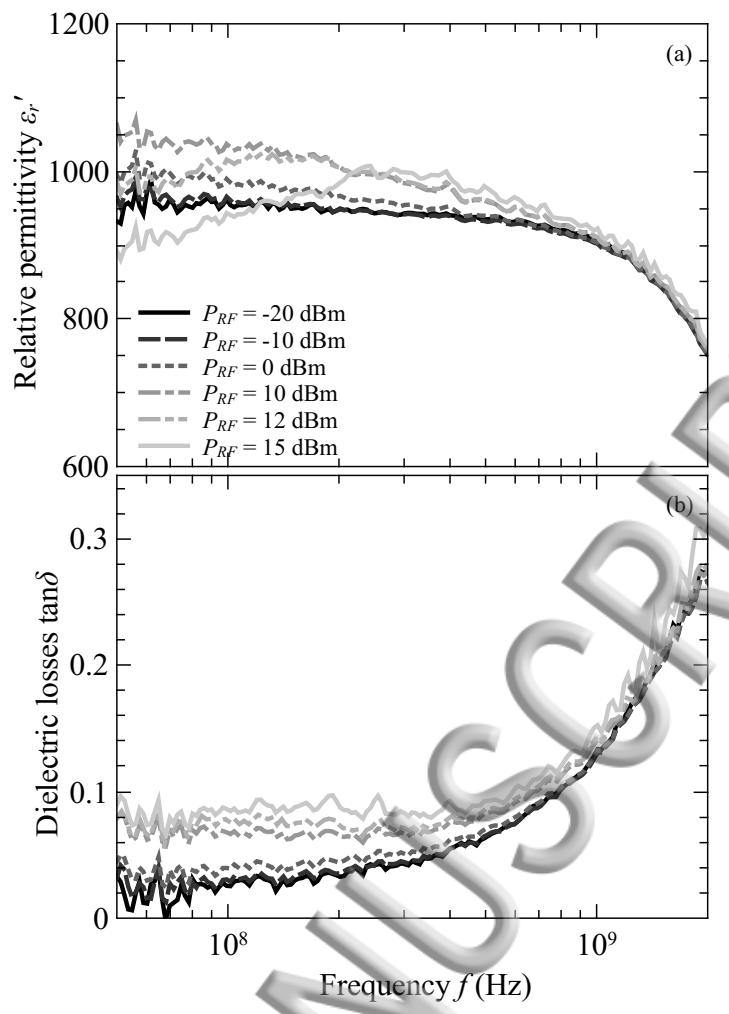

Figure 2: Relative permittivity (a) and dielectric losses (b) of the BST layer integrated in a MIM device, as a function of the frequency for different incident power, at $30^{\circ} \mathrm{C}$.

Fig. 2 shows the relative permittivity and the dielectric losses as a function of the frequency for different incident powers for a temperature of $30^{\circ} \mathrm{C}$. Up to $10 \mathrm{dBm}$ the relative permittivity increases with rising incident power, which is due to the domain wall pinning/unpinning contribution to the permittivity ${ }^{9,10}$. At $100 \mathrm{MHz}$, the permittivity grows from 955 to 1033, when the incident power increases from $-20 \mathrm{dBm}$ to $10 \mathrm{dBm}$, which represents an increase of $8 \%$. This indicates that a non-negligible part of the crystallites is in the ferroelectric phase. This behavior has been already shown for this material at lower frequencies ${ }^{19}$. However, the fraction of crystallites in the ferroelectric phase does not seem sufficient in order to have a hysteresis on the $\varepsilon_{r}^{\prime}\left(E_{D C}\right)$ loop.

The higher the frequency, the less an increase of the permittivity is visible. This comes from the driving field which stays very low even if the incident power increases, due to the diminution of the prefactor $\left|1+\Gamma_{C}\right|(5)$. Hence, the increase of the driving field is not sufficient to perceive the presence of the domain wall pinning/unpinning. To be able to observe their presence at frequencies higher than $1 \mathrm{GHz}$, a smaller device capacitance value would be needed.

The dielectric losses also increase when the incident power increases. Nevertheless, this varia- 


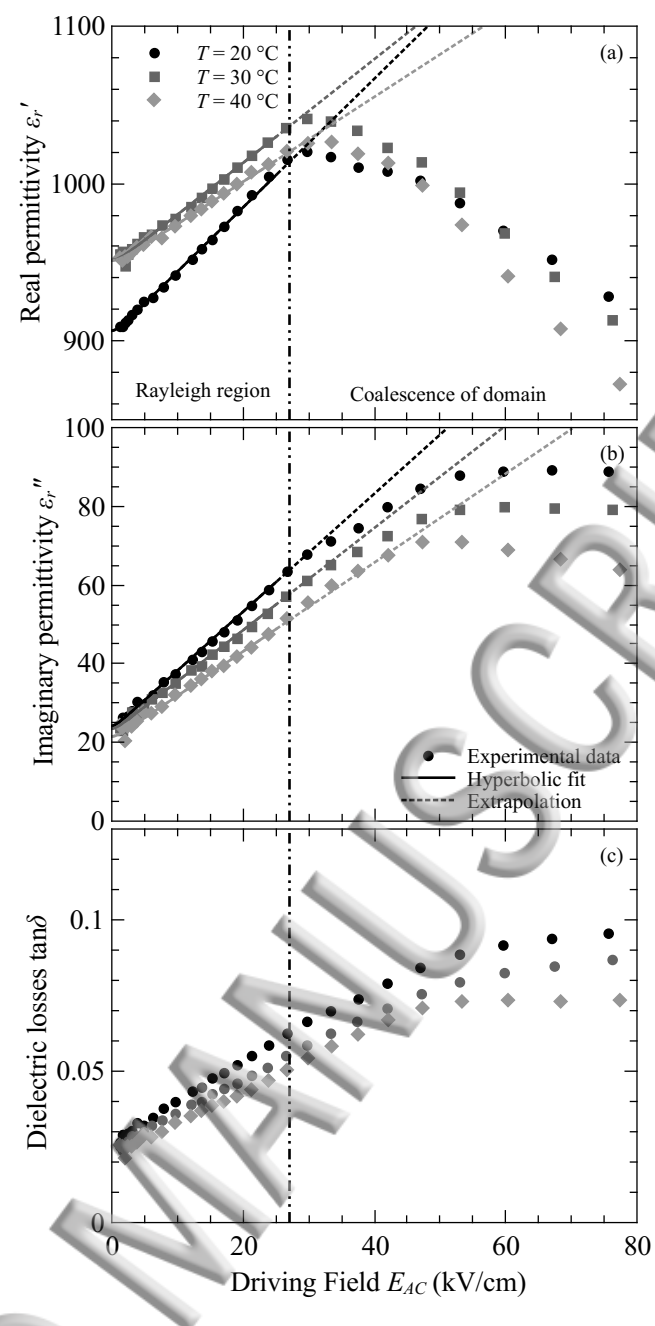

Figure 3: Real (a) and imaginary (b) parts of the relative permittivity and associated hyperbolic fits and the dielectric losses (c) as a function of the driving field for different temperatures, at $100 \mathrm{MHz}$.

tion is higher than for the permittivity since at $100 \mathrm{MHz}$, the losses increase from 0.025 to 0.066 , when the incident power varies from $-20 \mathrm{dBm}$ to $10 \mathrm{dBm}$, which represents an increase of $164 \%$ (compared to $8 \%$ for the permittivity). This reveals the very dissipative behavior of the domain wall pinning/unpinning and shows that this contribution should be reduced in order to limit the losses of the material influencing the overall behavior of a practical device.

Above $10 \mathrm{dBm}$ and for frequencies below $200 \mathrm{MHz}$, the driving field is sufficient to cause the saturation of the material which consists of a decrease of the permittivity. The losses increase since a part of the incident power (at $f_{0}$ ) is absorbed and converted into harmonics $\left(\text { at } k f_{0}\right)^{4}$. This saturation is not visible for higher frequencies since the prefactor $\left|1+\Gamma_{C}\right|$ becomes small and the 
Fig. 3 shows the real and imaginary parts of the permittivity and the dielectric losses as a function of the driving field for different temperature at a frequency of $100 \mathrm{MHz}$. The imaginary part of the permittivity has been calculated using $\varepsilon_{r}^{\prime \prime}=\varepsilon_{r}^{\prime} \tan \delta$. The driving field has been calculated using (5) for each temperature. For the three temperatures, the evolutions with the driving field are very similar showing that a non negligible part of the crystallites of the material presents a ferroelectric behavior showing the diffuse nature of the transition.

For driving fields, below $27 \mathrm{kV} / \mathrm{cm}$, the real and imaginary parts of the permittivity increase, due to the domain wall pinning/unpinning contribution, similarly to what have been noticed previously. The dielectric losses also increase showing the increase of the imaginary part is proportionally higher than for the real part, exhibits the dissipative behavior of the domain wall pinning/unpinning ${ }^{11-13}$. Again, this is due to the diffuse behavior of the transition. The presence of ferroelectric crystallites remains up to $40^{\circ} \mathrm{C}$ and possibly at higher temperature ${ }^{19}$ since an increase of the real part of the permittivity is well visible $(7.6 \%)$ even at $40^{\circ} \mathrm{C}$.

For driving fields above $27 \mathrm{kV} / \mathrm{cm}$, the real part of the permittivity decreases due to the coalescence of domains. This decrease of the permittivity comes from decrease of the number of domain. The driving field is sufficient to change the polarization state of the material. In this region, the permittivity diverges from the fit, showing that the hyperbolic law cannot be applied. The imaginary part of the permittivity still increases since a part of the power is absorbed to switch a part of the polarization. Since the imaginary part increases and the real part decreases, the dielectric losses (ratio between imaginary and real parts) become higher.

Rather stable dielectric losses are observed for driving fields higher than $60 \mathrm{kV} / \mathrm{cm}$. Different conflicting phenomena, influencing the real and the imaginary parts of the permittivity, may explain this behavior. As the driving field increases, losses also increase due to enhanced domain wall pinning/unpinning and a part of the power is absorbed to partially switch the polarization. In turn, polarization switching causes a diminution of the number of domains. As the contributions of domain wall vibration and pinning/unpinning to the complex permittivity is proportional to the number of domain walls ${ }^{20}$, a diminution of permittivity and dielectric losses is observed when global switching occurs. The observed "saturation" of the dielectric losses seems to be a compromise between these phenomena. It should be pointed out, that the variation of the permittivity and the losses can not be attributed to a heating effect as in ${ }^{4}$ since this would affect the dielectric properties in the whole frequency band, which is not the case here (Fig. 2). 
able I: Hyperbolic coefficients of the real and imaginary parts of the relative permittivity and associated dissipation factor for each contribution to the permittivity at a frequency of $100 \mathrm{MHz}$.

\begin{tabular}{|c|c|c|c|c|c|c|c|}
\hline \multirow{2}{*}{ Temperature } & \multicolumn{2}{|r|}{ Real part } & \multicolumn{2}{|c|}{ Imaginary part } & \multicolumn{2}{|c|}{ Dissipation factor } & \multirow{2}{*}{$\begin{array}{c}\text { Threshold field } \\
E_{t h}\end{array}$} \\
\hline & $\varepsilon_{r l}^{\prime}$ & $\varepsilon_{r-\text { rev }}^{\prime} \alpha_{r}^{\prime}(\mathrm{cm} / \mathrm{kV})$ & $\varepsilon_{r l}^{\prime \prime}$ & $\varepsilon_{r-r e v}^{\prime \prime} \alpha_{r}^{\prime \prime}(\mathrm{cm} / \mathrm{kV})$ & $m_{\varepsilon_{r l}}$ & $m_{\alpha_{r}}$ & \\
\hline $20^{\circ} \mathrm{C}$ & $900 \pm 2$ & $8 \pm 34.29 \pm 0.08$ & $23.2 \pm 0.7$ & $1 \pm 1 \quad 1.50 \pm 0.05$ & $0.027 \pm 0$ & $35 \pm 0.02$ & $1.9 \pm 0.6$ \\
\hline $30^{\circ} \mathrm{C}$ & $947 \pm 3$ & $6 \pm 5 \quad 3.3 \pm 0.2$ & $22.5 \pm 0.3$ & $1 \pm 1 \quad 1.30 \pm 0.02$ & $0.024 \pm 0$. & $39 \pm 0.03$ & $1.8 \pm 0.9$ \\
\hline $40^{\circ} \mathrm{C}$ & $946 \pm 2$ & $5 \pm 32.77 \pm 0.08$ & $20.5 \pm 1.6$ & $1 \pm 1 \quad 1.13 \pm 0.01$ & $0.022 \pm 0$ & $42 \pm 0.02$ & $1.8 \pm 0.9$ \\
\hline
\end{tabular}

The real and imaginary parts of the permittivity have been fitted using the hyperbolic law and the extracted coefficients are reported in the Table I. The fitting has been made using the Levenberg-Marquat algorithm which also gives the uncertainty on the extracted parameters $\left(\varepsilon_{r l}\right.$, $\varepsilon_{r-r e v}$ and $\alpha_{r}$ ). Using real and imaginary coefficients, it is possible to calculate the dissipation factor of all contributions $m_{\varepsilon}=\frac{\varepsilon^{\prime \prime}}{\varepsilon^{\prime}}$ with $\varepsilon$ corresponding to $\varepsilon_{r l}, \varepsilon_{r-r e v}$ and $\alpha_{r}$. Since the dissipation factor is the ratio between imaginary and real parts, its uncertainty has been calculated using the following formula:

$$
\delta m_{\varepsilon}=\left(\frac{\delta \varepsilon^{\prime}}{\varepsilon^{\prime}}+\frac{\delta \varepsilon^{\prime \prime}}{\varepsilon^{\prime \prime}}\right) m_{\varepsilon}
$$

$\delta \varepsilon^{\prime \prime}$ and $\delta \varepsilon^{\prime}$ are respectively the uncertainty associated the imaginary and real parts of the considered contribution. The precision on the extraction of the bulk values and the pinning/unpinning coefficients is quite good but the uncertainty is higher for the domain vibration contribution since the value is small compared to the bulk. As a consequence, the uncertainty on the dissipation factor associated to the bulk and pinning/pinning contributions are relatively low.

The real and imaginary parts follow well the hyperbolic law (full line). When the temperature increases, the slope of the real and imaginary parts versus the driving field, represented by the Rayleigh coefficient $\alpha_{r}$, decrease (Table I). This diminution indicates that the proportion of ferroelectric crystallites in the material decreases when the temperature increases, which has already been reported previously ${ }^{19}$. This decrease is progressive due to the polycristalline structure of the material. The parameters representing the domain vibration $\varepsilon_{r-r e v}^{\prime}$ are relatively hard to obtain since they are low compared to the lattice parameter (0.5\%). As a consequence, the evolution with the temperature is not easy to determine.

The dissipation factors for the domain wall contributions are one order of magnitude higher than the one associated to the lattice, confirming the extremely dissipative behavior ${ }^{11,12}$. When 
Publishithg temperature increases, the dissipation factor of the lattice contribution decreases which is coherent with a material going to the paraelectric phase. On the contrary, the dissipation factor for the domain wall pinning/unpinning slightly increases. This indicates that when the temperature increases, the domain wall motion is more dissipative. As the temperature increases and the ferroelectric behavior decreases, the polarization in the region/surrounding pinning sites can be reoriented at reasonable electric fields. This can be seen on the unpinning threshold field $\left(E_{t h}=\varepsilon_{r-\text { rev }}^{\prime} / \alpha_{r}^{\prime 10}\right)$ which seems to slightly decrease when the temperature increases, even if the precision on this parameter is quite low (Table I). Therefore, domain walls are more mobile due to unpinning at reasonable applied fields, leading to higher dissipative losses.

In this paper, the dielectric properties of a BST thin film have been studied in the microwave domain, as a function of the incident power and for different temperatures. When the incident power increases, the permittivity increases due to the domain wall pinning/unpinning contribution. A high increase of the dielectric losses is also noticed which is attributed to the very dissipative behavior of the domain wall pinning/unpinning. This proves the diffuse nature of the ferro/paraelectric transition which needs to be considered for the characterization or the use of the material into a device. When the temperature increases, the domain wall pinning/unpinning contribution decreases indicating that the ferroelectric nature of the material "progressively vanishes". This clearly shows that the characterization of functional material needs to be made for the foreseen power since the non-linearities of the ferroelectrics are present even at low and high driving fields.

The authors gracefully acknowledge L. Trupina and L. Nedelcu from the National Institute of Materials Physics (Romania) for the iridium bottom electrode deposition. The authors from XLIM and IRCER acknowledge the financial support from the France Region Limousin under the H2020 European project MASTERS (M-ERA.NET call).

\section{REFERENCES}

${ }^{1}$ K. Nadaud, R. Gillard, E. Fourn, H. Gundel, and C. Borderon, in Loughborough Antennas and Propagation Conference (2014) pp. 214-217.

${ }^{2}$ A. Ghalem, F. Ponchel, D. Remiens, and T. Lasri, in Proc. IEEE Int. Symp. Applicat. Ferroelectrics (2013) pp. 252-256.

${ }^{3}$ J. Hai, M. Patterson, D. Brown, Z. Chenhao, P. KuanChang, G. Subramanyam, D. Kuhl, 
Publishing. Leedy, and C. Cerny, IEEE Transactions on Antennas and Propagation 60, 3111 (2012).

${ }^{4}$ P. Rundqvist, A. Vorobiev, E. Kollberg, and S. Gevorgian, Journal of Applied Physics 100, 1 (2006).

${ }^{5}$ Y.-K. Yoon, D. Kim, M. G. Allen, J. S. Kenney, and A. T. Hunt, IEEE Transactions on Microwave Theory and Techniques 51, 2568 (2003).

${ }^{6}$ H. Katta, H. Kurioka, and Y. Yashima, in 2006 IEEE MTT-S International Microwave Symposium Digest (2006) pp. 564-567.

${ }^{7}$ K. Nadaud, C. Borderon, R. Renoud, A. Ghalem, A. Crunteanu, L. Huitema, F. Dumas-Bouchiat, P. Marchet, C. Champeaux, and H. W. Gundel, Applied Physics Letters 109, 262902 (2016).

${ }^{8}$ K. Nadaud, C. Borderon, R. Renoud, A. Ghalem, A. Crunteanu, L. Huitema, F. Dumas-Bouchiat, P. Marchet, C. Champeaux, and H. W. Gundel, Applied Physics Letters 110, 212902 (2017).

${ }^{9}$ D. V. Taylor and D. Damjanovic, Journal of Applied Physics 82, 1973 (1997).

${ }^{10}$ C. Borderon, R. Renoud, M. Ragheb, and H. W. Gundel, Applied Physics Letters 98, 112903 (2011).

${ }^{11}$ J. E. García, R. Pérez, and A. Albareda, Journal of Physics: Condensed Matter 17, 7143 (2005).

${ }^{12}$ J. E. García, R. Pérez, D. A. Ochoa, A. Albareda, M. H. Lente, and J. A. Eiras, Journal of Applied Physics 103, 054108 (2008)

${ }^{13}$ K. Nadaud, C. Borderon, R. Renoud, and H. W. Gundel, Journal of Applied Physics 119, 114101 (2016).

${ }^{14}$ A. Ghalem, M. Rammal, L. Huitema, A. Crunteanu, V. Madrangeas, P. Dutheil, F. DumasBouchiat, P. Marchet, C. Champeaux, L. Trupina, L. Nedelcu, and M. G. Banciu, IEEE Microwave and Wireless Components Letters 26, 504 (2016).

${ }^{15}$ V. V. Lemanov, E. P. Smirnova, P. P. Syrnikov, and E. A. Tarakanov, Phys. Rev. B 54, 3151 (1996).

${ }^{16}$ A. Ghalem, L. Huitema, A. Crunteanu, M. Rammal, L. Trupina, L. Nedelcu, M. G. Banciu, P. Dutheil, C. Constantinescu, P. Marchet, F. Dumas-Bouchiat, and C. Champeaux, Journal of Applied Physics 120, 184101 (2016).

${ }^{17}$ G. A. Rossetti, L. E. Cross, and K. Kushida, Applied Physics Letters 59, 2524 (1991).

${ }^{18}$ Z. Ma, A. J. Becker, P. Polakos, H. Huggins, J. Pastalan, H. Wu, K. Watts, Y. H. Wong, and P. Mankiewich, IEEE Transactions on Electronics Devices 45, 1811 (1998).

${ }^{19}$ L. M. Garten, P. Lam, D. Harris, J.-P. Maria, and S. Trolier-McKinstry, Journal of Applied Physics 116, 044104 (2014). 
Publishifig Boser, Journal of Applied Physics 62, 1344 (1987).

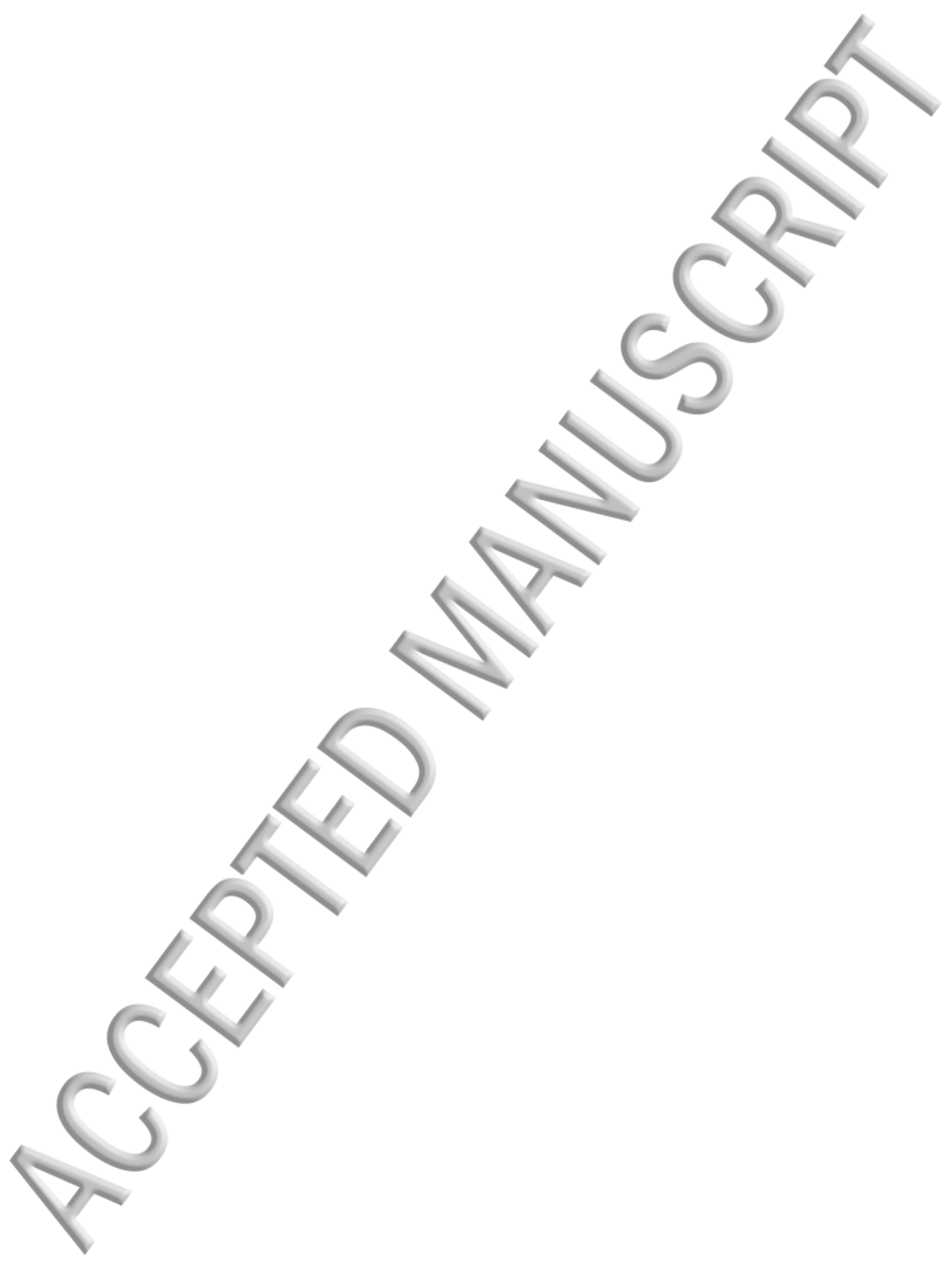




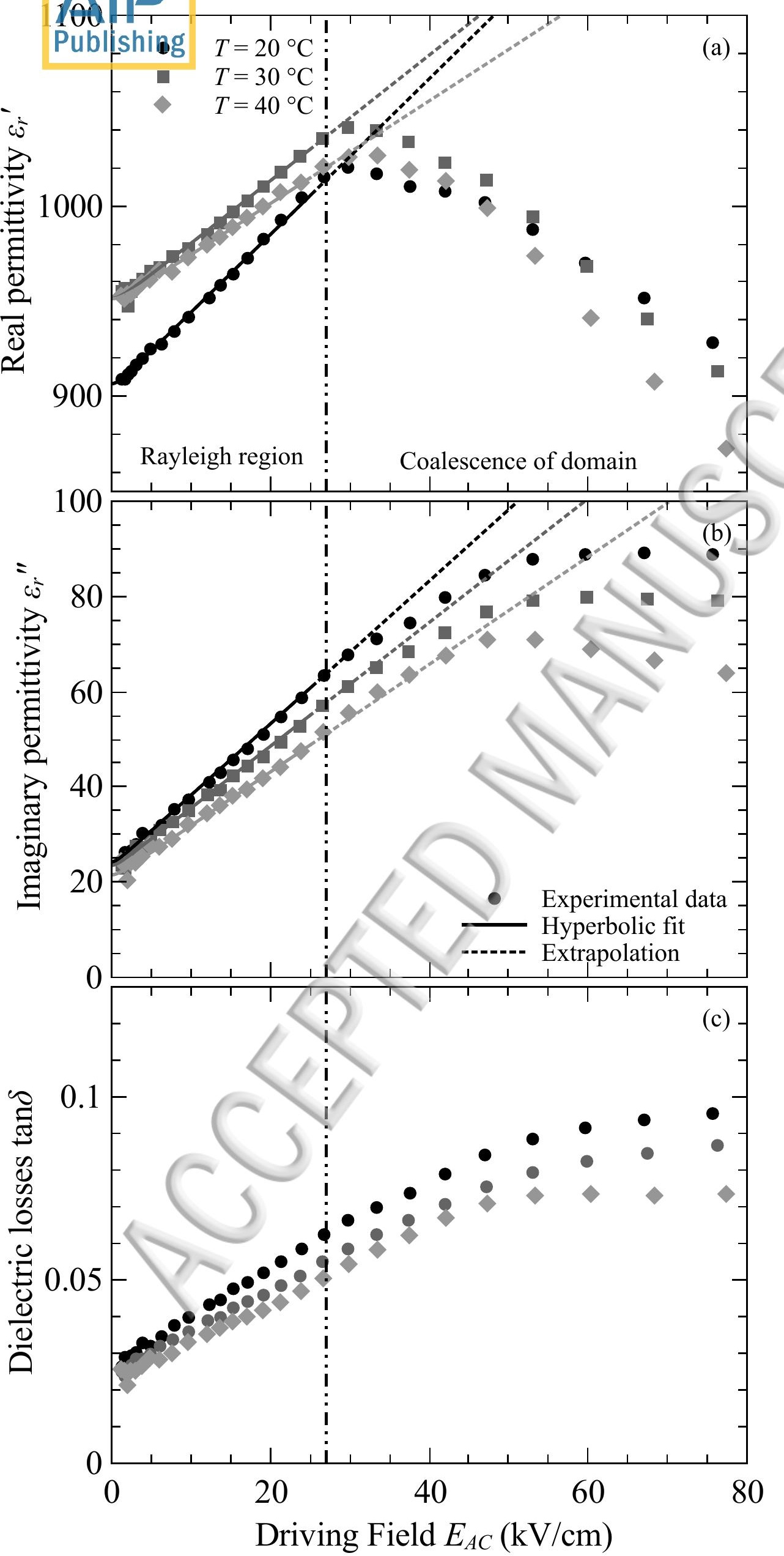

